\title{
TREE-BASED MULTICAST KEY AGREEMENT
}

\author{
Jörg Schwenk, Tobias Martin and Ralf Schaffelhofer \\ T-Nova Deutsche Telekom Innovationsgesellschaft $\mathrm{mbH}$ \\ Technologiezentrum \\ Am Kavalleriesand 3, 64295 Darmstadt, Germany \\ \{joerg.schwenk, tobias.martin, ralf.schaffelhofer\}@t-systems.de
}

\begin{abstract}
We present an analysis of a multicast key agreement scheme based on the iterated Diffie-Hellman key agreement protocol and binary trees. This scheme is extremely efficient for deleting single members from the group and joining of groups. It does not need a group moderator, and is self-synchronizing.
\end{abstract}

Keywords: IP Multicast, group key agreement, Diffie-Hellmann key agreement

\section{INTRODUCTION}

IP Multicast is an efficient way to distribute content to many recipients. It can be used for streaming audio/video, or for video conferencing over the internet.

If IP Multicast is used to distribute content in a unidirectional way (e.g. streaming audio), then the source of the content can play a special role in the IP Multicast group: It can act as the group moderator who accepts or rejects new group members, and distributes the encryption keys to them. Therefore, key management can be handled in a centralized manner.

In other applications like video conferencing, there is no natural choice for the role of the group moderator. In contrast, if there is a group moderator, then he can not leave a video conference without terminating it.

In this paper we analyze a self-synchronizing protocol for adding and deleting group members, and for the joining of groups. In these protocols, there is no need for a group moderator. The members of a group are associated with the leaves of a binary tree, and iterated Diffie-Hellman key agreement as described in [BW98] is used to compute the group key.

A lot of research has been done to extend [DH76] to more than two participants ([AST98], [BD94], [BW98], [ITW82], [STW96], [STW98]). We will briefly review this research in section 3 . However, most of the proposed protocols assume that each participant knows to whom he has to send the intermediate 
key result. These assumptions do not hold for IP multicast, which is a receiverbased protocol. This means that at IP level, the sender does not have to know the list of recipients of a multicast group. A short introduction to IP multicast will be given in Section 2.

Section 3 gives an overview of related articles and summarizes their results. In section 4 we discuss the Burmester-Desmedt [BD94] in detail and give an overall view on its complexity, specifically on the complexity of member deletion. In section 5 the iterated Diffie-Hellmann protocol is defined which is used in section 6 where the tree-based key agreement protocol is introduced. In that section an approach is given together with explicit algorithms and proofs on their complexity. Finally, in section 7 we state some open problems for multicast security on group key agreement protocols.

\section{IP MULTICAST}

IP multicast is a concept used to reduce traffic on IP networks: If data is sent to many recipients with the normal IP unicast mechanism, many copies of the same data are sent, and the IP network is flooded with redundant data. This problem especially arises for bandwidth-consuming applications like audio or video transmissions over the internet.

IP multicast solves this problem in the following way:

- First, a unique IP address is assigned to the multicast group: the multicast address.

- If a user wants to join the multicast group, he has to send a request to his multicast-enabled LAN-Router to be included in the group.

- Based on these requests, the intermediate routers build up a spanning tree through the IP network. Multicast data is only routed along this spanning tree. This guarantees that no unnecessary copies of the content are sent in a network segment.

The design of routing protocols that construct this spanning tree is the most challenging task of IP multicast. More information about IP Multicast can be found at [Multicast].

This short introduction should be sufficient to motivate the following criteria for selecting suitable key agreement protocols.

- Efficiency. The selected protocols should be efficient for small and for large groups of participants.

- Receiver based key agreement. The sender of a key agreement message should only have to know as little as possible about the receiver set of 
the message. Ideally, he should only have to know that there is a set of receivers out there, and the cardinality of this set.

- Easy addition of new group members. Following [STW98], we distinguish between Initial Key Agreement (IKA) and Auxiliary Key Agreement (AKA) operations. For the addition of new group members, AKA operations should be available which are more efficient than performing an IKA again.

- Easy deletion of group members. It should also be possible to delete group members via an efficient AKA.

- Easy addition/deletion of subgroups. AKA operations should be available for inserting/deleting whole subgroups of participants.

- Message Broadcast. The selected protocols should aim at minimizing the amount of data exchanged during key establishment by making use of the IP multicast mechanism.

- Equivalence with Diffie-Hellman Key exchange. The security of the proposed protocols should be equivalent to the security of the DiffieHellman Key exchange.

The criteria described above are difficult to achieve. The proposed protocols only partially fulfil them. Nevertheless each of the protocols proposed has the properties of perfect forward secrecy and is contributory.

Definition 1 A key agreement protocol provides perfect forward secrecy if disclosure of long-term keying material does not compromise the secrecy of the exchanged messages from earlier sessions.

Definition 2 A key agreement protocol is contributory if each party contributes to the key and thus guarantees its freshness.

\section{RELATED WORK}

Research on group key agreement starts with the classical paper of Ingemarsson, Tang and Wong [ITW82]. The authors examine the possibility of using symmetric functions in the private keys of the participants to derive the group key. Their main result is that a symmetric function must have maximal degree in order to be secure. E.g. if A, B and C want to generate a common key $k$ from their private keys $a, b$ and $c$, the function $k=g^{a b c}$ is secure, while $k=g^{a+b+c}$ and $k=g^{a b+a c+b c}$ are insecure. Since a symmetric function would be desirable to implement a true receiver based key agreement, this result means that for generating a common key amongst $\mathbf{n}$ participants, $n$ rounds of communication are necessary. 
By modifying the ideas from [ITW82], Burmester and Desmedt introduced what is up to now the most elegant and efficient group key agreement protocol [BD94]. They use cyclic functions instead of symmetric ones, and in a broadcast scenario such as IP multicast, key agreement for $n$ participants is done within two rounds and $2 n$ messages. However, they assume that a cyclic structure on the set of participants is (at least partly) known to each participant. There is an efficient AKA scheme for including a new member in the group which requires only four messages to be broadcast [STW98]. On the other hand, at least 1.5n messages are neccessary to exclude a member (see Section 4).

In a second paper [BD96] the authors propose even more efficient schemes for group key exchange. However, these protocols are not contributory and will therefore not be considered in this paper. Three new protocols for group key agreement have been proposed by Steiner, Tsudik and Waidner [STW96] which assume a linear ordering on the set of participants. Subsequently, the authors have extended these protocols to AKA schemes [STW98], and, with the help of G. Ataniese [AST98], to include authentication. Our work complements these approaches.

A very interesting paper on the communication complexity of group key agreement schemes has recently been published by Becker and Wille [BW98]. The authors give upper and lower bounds on the number of messages, exchanges and rounds, and they give examples for protocols with a minimum number of exchanges and rounds in the non-broadcast scenario. In these examples, they follow a tree-based approach, and they prove the equivalence of the security of a tree based protocol with Diffie-Hellman key exchange.

We do not follow an approach described by A. Ballardie [B96] which delegates security functions to routers: We believe that all security functions should be controlled by the participants.

The parameters of our schemes compared to the Burmester-Desmedt [BD94] scheme are summarized in the following table. The number of rounds is 2 for all protocols except for our TBKA. The rows correspond to the actions of initial key agreement (IKA), adding one participant (Add 1), deleting one participant (Delete 1) and joining two groups.

\begin{tabular}{|l|c|c|c|}
\hline & [BD94] & \multicolumn{2}{c|}{ Tree Based Key Agreement } \\
& messages & rounds & messages \\
\hline IKA & $2 n$ & $\log _{2} n$ & $2 n-2$ \\
\hline Add 1 & 4 & $\log _{2} n$ & $2 \log 2 n$ \\
\hline Delete 1 & $1.5 n$ & $\log 2 n$ & $2 \log 2 n$ \\
\hline Join Groups & $2 k+3$ & 1 & 2 \\
\hline
\end{tabular}




\section{DELETING PARTICIPANTS IN THE BURMESTER-DESMEDT PROTOCOL}

In [BD94], Burmester and Desmedt described a very efficient group key agreement protocol based on cyclic polynomials. However, this protocol does not support any efficient AKA protocol for member deletion.

The Burmester-Desmedt protocol consists of two broadcast rounds and a final computation. It works for any finite cyclic group $(G, \cdot)$ of order $q$ where the discrete logarithm is hard to compute.

- Let $g$ be a generator of the group $G$.

- In the first round, each participant $P_{i}, i=1, \ldots, n$, selects a random number $r_{i}$ and broadcasts $z_{i}=g^{r_{i}}$.

- In the second round, $P_{i}$ broadcasts $X_{i}=\left(z_{i+1} / z_{i-1}\right)^{r_{i}}=g^{r_{i+1} r_{i}-r_{i} r_{i-1}}$ (all indices modulo $n$ ).

- Having received all $X_{j}, j \neq i, P_{i}$ can compute the group key

$$
\begin{aligned}
K & =\left(z_{i-1}\right)^{n r_{i}} \cdot X_{i}^{n-1} \cdot X_{i+1}^{n-2} \cdots X_{i-2} \\
& =g^{r_{i} r_{i-1}+r_{i+1} r_{i}+\cdots+r_{i-2} r_{i-3}+r_{i-1} r_{i-2}} .
\end{aligned}
$$

Lemma 1 In the Burmester-Desmedt protocol, any participant can compute all group elements of the form $g^{r_{i} r_{i+1}}$.

Proof. Let w.l.o.g. consider participant $P_{1}$. He knows $r_{1}$ and $z_{2}=g^{r_{2}}$, therefore he can compute $g^{r_{1} r_{2}} . P_{1}$ also knows $X_{2}=g^{r_{2} r_{3}-r_{1} r_{2}}$ and computes

$$
X_{2} \cdot g^{r_{1} r_{2}}=g^{r_{2} r_{3}} .
$$

In general, if $P_{1}$ knows $g^{r_{j-1} r_{j}}$ and $X_{j}$, he can also compute $g^{r_{j} r_{j+1}}$. This proves the lemma.

Theorem 1 In any member exclusion protocol for the Burmester-Desmedt key agreement scheme where two consecutive random numbers $r_{i}$ and $r_{i+1}$ remain unchanged, the excluded member can compute the new group key.

Proof. Let

$$
\tilde{K}=g^{\tilde{r}_{1} \tilde{r}_{2}+\tilde{r}_{2} \tilde{r}_{3}+\cdots+\tilde{r}_{i-1} r_{i}+r_{i} r_{i+1}+r_{i+1} \tilde{r}_{i+2}+\cdots+\tilde{r}_{n-1} \tilde{r}_{n}}
$$

be the new group key after participant $P_{1}$ has been excluded. Following lemma 1, $P_{1}$ knows the group element $g^{r_{i} r_{i+1}}$. From

$$
\tilde{X}_{i+1}=g^{r_{i+1} \tilde{r}_{i+2}-r_{i} r_{i+1}}
$$


$P_{1}$ can compute $g^{r_{i+1} \tilde{r}_{i+2}}$ and can thus successively compute all factors of the new key. By multiplying them, he gets the new key.

Corollary 1 To exclude a member from a group with the Burmester-Desmedt protocol, at least $1.5 n$ broadcast messages are needed.

Proof. As a result of theorem 1, at least every other random number has to be changed. This means that at least half of the $z_{j}$, and all the $X_{j}$ have to be changed and resent.

\section{ITERATED DIFFIE-HELLMAN}

The basic idea behind the iterated Diffie-Hellman protocol is to use the key generated in one round of the protocol as a random input for the next round. This idea was first published in [BW98], where it was used to provide examples for key exchange protocols with a minimal number of rounds.

Definition 3 Let $G$ be a finite cyclic group of order $q$, let $g$ be a generator of $G$ and let $\varphi: G \rightarrow \mathbf{Z}_{q}$ be a bijection. The $n$ participants are represented by the leaves of a binary tree $T$ of depth $m$ with the following properties:

- The levels of $T$ are numbered from 0 to $m-1$, starting at the root with 0

- The nodes in level $i$ are numbered from 0 to $2^{i}-1$. We write $N_{j, i}$ for the $j$-th node in level $i$

- A group element $k_{j, i}$ is associated with each $N_{j, i}$

Then the iterated Diffie-Hellman key for $n$ participants is the group element $k_{0,0}$ which is computed as

- $k_{j, i}$ is randomly chosen from $\mathbf{Z}_{q}$ if $N_{j, i}$ is a leaf of $T$

- $k_{j, i}=g^{\varphi\left(k_{2 j, i+1}\right) \cdot \varphi\left(k_{2 j+1, i+1}\right)}$ if $N_{j, i}$ is not a leaf of $T$ and thus has two successors ${ }^{1}$

For simplicity of notation we assume in the following $G=\mathbf{Z}_{p}^{*}$, the original choice of the group $G$ in [DH76]. Then the bijection $\varphi$ is $\varphi=i d-1$. For other choices of $G$, e.g. as a prime order subgroup of a larger $\mathbf{Z}_{r}$, and for elliptic curves, suitable (efficiently computable) bijections have to be found.

\footnotetext{
${ }^{1}$ Note that $N_{j, i}$ must have two successors if it is not a leaf: if it had just one we could remove $N_{j, i}$ from the tree and connect its predecessor with its successor directly
} 

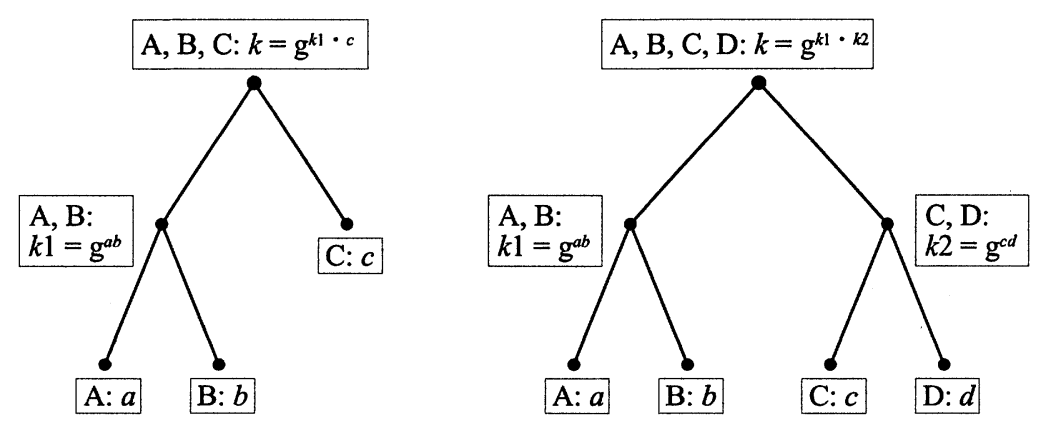

Figure 1 Tree based key agreement with 3 or 4 participants.

\section{TREE BASED KEY AGREEMENT}

The idea behind tree based key agreement is to use a key hierarchy to build successively larger groups by applying a Diffie-Hellman key exchange between the groups. Our scheme should not be mixed up with the tree based scheme from [BD94], where a Diffie-Hellman key agreement is only used to establish secure channels along the edges of the tree.

The advantage of a tree based scheme is that each of the $n$ participants only has to perform at most $\log n \mathrm{DH}$-operations to establish a common key. Adding or removing a participant to or from a group requires $\log n \mathrm{DH}$ operations only for the immediate neighbors of the added or removed node, and joining and dividing groups is even easier.

\subsection{INITIAL KEY AGREEMENT (IKA)}

We assume that the group is structured as a binary tree, where the participants are represented by the leaves. If two nodes have a common predecessor, this means that these two nodes have to perform a Diffie-Hellman key agreement to generate a common key. Figure 1 illustrates this process for 3 and 4 participants. Let $P_{1}, \ldots, P_{n}$ be the participants who want to agree on a group key, and let $T$ be a binary tree where the leaves are associated with the participants, and where each participant knows all vertices from his leaf to the root of the tree. Let $\operatorname{par}(A)$ denote the parent of the vertex $A, R$ the root of the tree and level $(A)$ the level of vertex $A$, i.e. the distance of vertex $A$ from the root $R$, with $\operatorname{level}(R)=0$.

1 Each participant $X$ chooses a random number $x$ and broadcasts

$$
\left(X, \operatorname{par}(X), \operatorname{level}(X), g^{x-1} \bmod p\right)
$$


$2 X$ waits until he receives a message

$$
(Y, \operatorname{par}(Y), \operatorname{level}(Y), h)
$$

with $\operatorname{par}(X)=\operatorname{par}(Y)$ (i.e. level $(X)=\operatorname{level}(Y))$ and computes

$$
k:=h^{x-1}=\left(g^{y-1}\right)^{x-1} \quad(\bmod p)
$$

3 For some order (e.g. alphabetically) compare the identities $X$ and $Y$ :

- If $Y<X, X$ becomes passive and only listens to the further multicast transmissions.

- Else $X$ broadcasts the message

$$
\left(\operatorname{par}(X), \operatorname{par}(\operatorname{par}(X)), \text { level }(\operatorname{par}(X)), g^{k-1} \bmod p\right) .
$$

4 This process is repeated until two messages with level 1 have been exchanged.

This algorithm can be described more precisely in pseudocode notation for one participant $P_{i}$. We can condense all information about the location of a vertex in the tree in a binary string which we call VID (Vertex ID). In our notation, the root has $V I D=1$ and when traversing the tree we add a 0 each time we go to the left and a 1 each time we go to the right. The Vertex ID for participant $B$ in both trees of figure 1 is VID $=101$.

The VID can be used very efficiently in our pseudocode example given below. If we treat the binary string as a number the following holds: for a vertex $X$ with $V I D_{X}$ we have $\operatorname{VID} \operatorname{par}(X)=V I D_{X} \div 2$ (where $\div$ denotes integer division) and $\operatorname{level}(X)=\left\lfloor\log _{2} \operatorname{VID}\right\rfloor$.

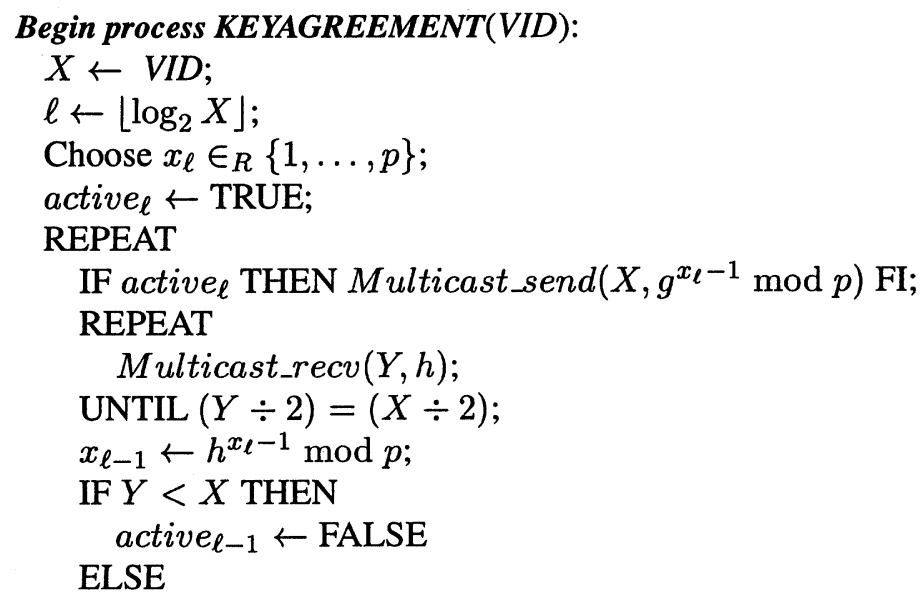




$$
\begin{aligned}
& \quad \text { active }_{\ell-1} \leftarrow \text { TRUE } \\
& \text { FI; } \\
& X \leftarrow X \div 2 \\
& \ell \leftarrow \ell-1 ; \\
& \text { UNTIL } X=1 \\
& k \leftarrow x_{0}
\end{aligned}
$$

End process.

\subsection{AUXILIARY KEY AGREEMENT (AKA)}

The AKA algorithm is used when an IKA has already been performed and e.g. a participant is going to be added as a leaf of the tree. The number of messages which have to be broadcast can be reduced since the value $k_{j, i}$ of the highest node of every subtree, which does not include the new member, has not to be changed.

Thus the AKA algorithm starts at the leaf where a member has been added or removed. An invalidate flag is set for every node which lies on the path from this leaf to the root. Key exchange messages are only broadcast for the nodes which have been invalidated. The members which were active during the IKA for these nodes reuse their stored values.

During auxiliary key agreement the following process has to be executed by every participant with vertex ID VID. The parameter valid indicates wether the corresponding node has been invalidated or not:

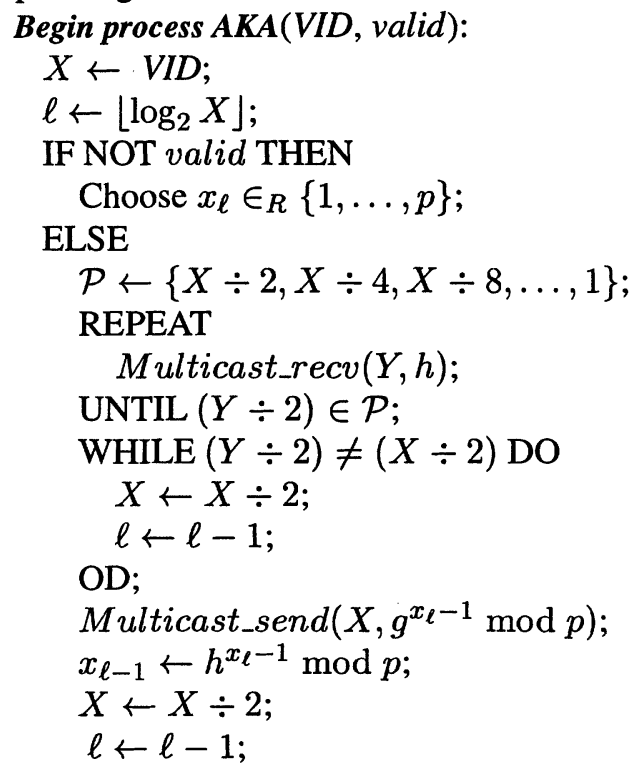

FI; 


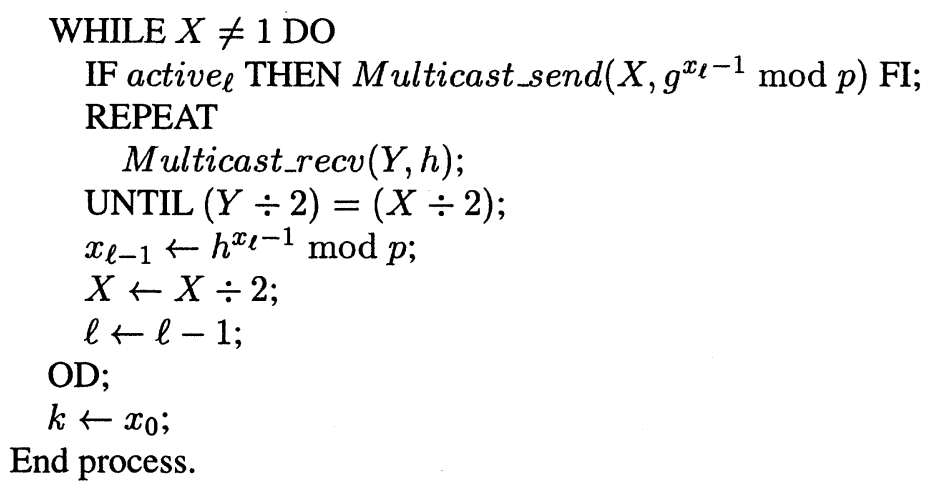

Theorem 2 The TBKA is contributory and has the perfect forward secrecy property. Forn participants, key agreement can be done within $\left\lceil\log _{2} n\right\rceil$ rounds, sending $2 n-2$ broadcast messages, with a maximum workload of $\left\lceil\log _{2} n\right\rceil$ exponentiations per participant.

Proof. For a proof of the equivalence with the DH key agreement see [BW98]. It is easy to see that the protocol is contributory, and it has the PFS property as long-term keys are only used for authentication. The complexity results from the fact that it is always possible to represent $n$ participants as leaves of a binary tree of depth at most $\left\lceil\log _{2} n\right\rceil$. For each vertex in this tree that is not a leaf, two messages have to be sent, so the number of messages is at most $2(n-1)$.

Remark 1 The security of the TBKA protocol can be proven along the lines of [BW98].

\subsection{ADDING A PARTICIPANT}

The assumption that the set of participants has a tree structure may seem rather restrictive, but one should keep in mind that this structure can be build up successively as the participants join the group. To guarantee that the tree is filled breadth first, the level number of each node can be used.

A new participant sending out a request to join the group would be attached to a leaf which has minimal level number in the tree. Figure 2 illustrates this algorithm.

The participant who wants to join the group executes the following process:

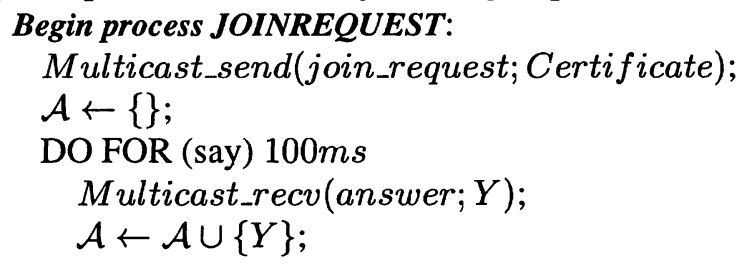




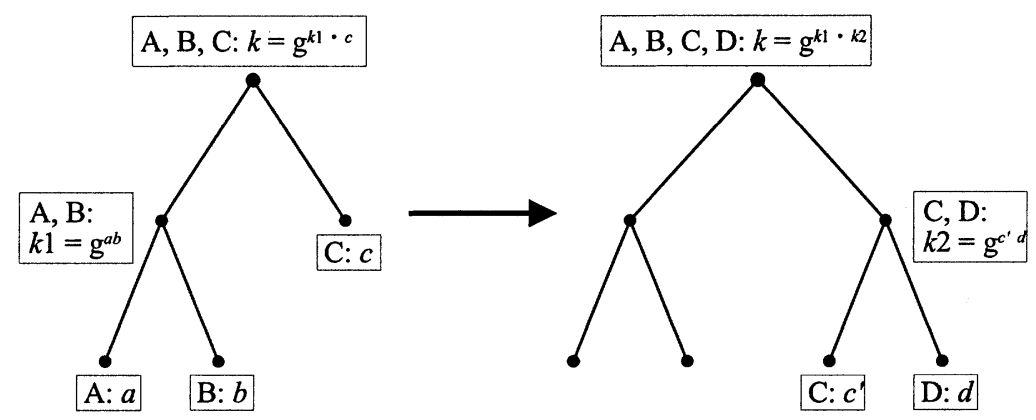

Figure 2 Adding a participant to a group. The new participant $D$ is added to the leaf with minimal level number. Only the computations shown on the right side have to be performed again.

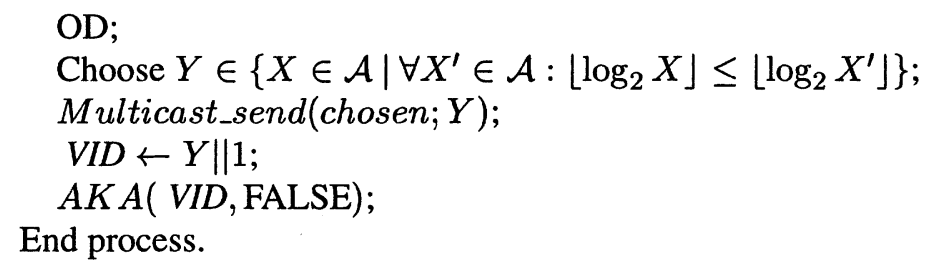

OD;

Choose $Y \in\left\{X \in \mathcal{A} \mid \forall X^{\prime} \in \mathcal{A}:\left\lfloor\log _{2} X\right\rfloor \leq\left\lfloor\log _{2} X^{\prime}\right\rfloor\right\}$;

Multicast_send(chosen; $Y)$;

$V I D \leftarrow Y \| 1$

$A K A($ VID, FALSE);

End process.

All other participants, who are already group members, have to perform the following actions once they receive the join request message:

Begin process JOINANSWER(VID):

level $\leftarrow\left\lfloor\log _{2}\right.$ VID $\rfloor$;

active $\leftarrow$ TRUE;

Multicast_recv(join_request;Certificate);

DO FOR level $\cdot 10 \mathrm{~ms}$

ON Multicast_recv(answer; $Y$ ) DO

IF $\left\lfloor\log _{2} Y\right\rfloor<$ level THEN active $\leftarrow$ FALSE FI; OD;

OD;

IF active THEN Multicast_send(answer; VID) FI;

Multicast_recv(chosen; $X)$;

IF $X=V I D$ THEN

$V I D \leftarrow V I D \| 0 ;$

$A K A$ ( VID, FALSE);

ELSE

$A K A$ (VID, TRUE);

FI;

End process. 


\subsection{REMOVING A PARTICIPANT}

The process of removing a participant from a group is the inverse process of adding: The two leaves of the removed participant and its immediate neighbor are removed from the tree, and the neighbor is assigned the new leaf, i.e. the VID of his former parent. His level number thus decreases by 1 .

The member who is going to be removed performs the following steps:

Begin process REMOVEREQUEST(VID):

REPEAT

Multicast_send(remove_request; VID);

UNTIL Multicast_recv(remove_acknowledge);

End process.

All other members of the group receive the request for removal and act to the following rules:

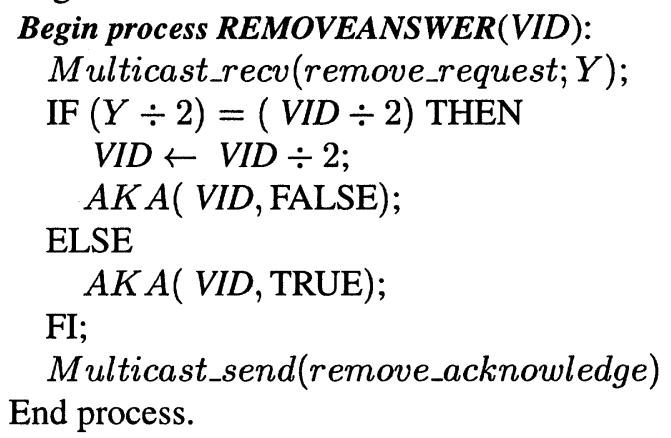

An advantage of our approach is that a former group member is able to prove his inability of participating in the future communication once he has sent a removal request and the subsequent AKA has been performed.

\subsection{JOINING AND SPLITTING GROUPS}

Groups can easily be joined by adding a new root node as the predecessor of the two original roots. Splitting of groups is difficult, except when the two groups are separated by a single node, i.e. when removing the node results in two tree structures which contain exactly (or up to small differences) the two subgroups. In all other cases a completely new IKA has to been performed.

\section{OPEN PROBLEMS}

Authentication of new group members in case that there is no group moderator is a difficult task. It can either be solved by using some predefined rules, e.g. that new participants with a valid certificate of a trusted certification authority will always be accepted, or some kind of voting is needed within the group. 
Authentication of a removal request is also a problem, especially if the removal request does not come from the participant himself.

Solving this problem is a challenging task to implement some kind of democracy for IP multicast in contrast to the centralized approach using a group moderator.

\section{References}

[AST98] G. Ateniese, M. Steiner and G. Tsudik: Authenticated group key agreement and friends, 5th ACM Conference on Computer and Communication Security, November 1998.

[B96] A. Ballardie: Scalable multicast key distribution, IETF RFC 1949, May 1996

[BW98] C. Becker and U. Wille, Communication complexity of group key distribution, ACM Conference on Computer and Communication Security, November 1998.

[BD94] M. Burmester and Y. Desmedt, A secure and efficient conference key distribution system, Eurocrypt'94, Springer LNCS, pp. 275288.

[BD96] M. Burmester and Y. Desmedt, Efficient and secure conference key distribution, Cambridge Workshop on Security Protocols, Springer LNCS 1189, pp 119-129 (1996).

[DH76] W. Diffie und M. Hellman: New Directions in Cryptography, IEEE Transactions on Information Theory, IT-22(6):644-654, November 1976

[HMR96] H. Harney, C. Muckenhirn and T. Rivers, Group key management protocol ( $\mathrm{gkmp}$ ) architecture, IETF Draft, 1996.

[ITW82] I. Ingemarsson, D. Tang and C. Wong, A conference key distribution system, IEEE Transactions on Information Theory, September 1982.

[Multicast] The MBONE Information Web, http://www. mbone.com.

[SMS98] J. Schwenk, T. Martin, R. Schaffelhofer, Verfahren zum Etablieren eines gemeinsamen kryptographischen Schlüssels für $n$ Teilnehmer, Patentanmeldung DE 19847941 A 1, 1998.

[STW96] M. Steiner, G. Tsudik and M. Waidner, Diffie-Hellman key distribution extended to groups, ACM Conference on Computer and Communication Security, pp 31-37, March 1996.

[STW98] M. Steiner, G. Tsudik and M. Waidner, CLIQUES: A new approach to group key agreement, IEEE International Conference on Distributed Computing Systems, May 1998. 